\title{
Exploration on Thermal Shock Mechanical Properties of Ceramic Materials
}

\author{
Yanxia Wang, Tiantian Sun, Qinghui Shang, Yongli Bao, Xiaomin Ma
}

School of Traffic and Vehicle Engineering, Shandong University of Technology, 255049, ZiBo, China.

E-mail: wangyx200@163.com

\begin{abstract}
Keywords: Fatigue failure; engineering mechanic experimental; engineering mechanic teaching;
\end{abstract} ceramic materials; elastic modulus; bending strength.

\begin{abstract}
Fatigue failure is very common phenomenon in engineering field, and fatigue problem is more and more prominent with the development of modern social production to high speed, high temperature, high strength load. In process of college undergraduate course graduation design and innovative experiments, this paper studies elastic modulus and bending strength change rule curve of the mullite ceramic specimens under different factors such as temperature difference, thermal shock times and different cooling medium. The paper studies the effects of different heat shock factors influence on the mechanical properties of mullite ceramics, and determines the thermal shock damage degree of ceramics materials, and then cultivate students the research ideas and research methods of fatigue failure.
\end{abstract}

\section{Introduction}

With the development of modern science and technology, materials are puts forward high demands on the departments such as space technology, industrial, energy, transportation and so on. Materials are required having high temperature resistance, erosion resistance, good thermal shock, heat preservation, oxidation resistance, excellent comprehensive performance. High temperature structure ceramic material has a series of excellent performance such as high temperature resistance, scouring resistance, corrosion resistance, high hardness, high wear resistance, high strength and oxidation resistance, so ceramic material is the focus and hotspot in the research of the scientific research workers[1-5].

In the current engineering mechanics course, the fatigue, especially the thermal fatigue, almost is not involved due to the limitation of school hours, The students almost have not fatigue failure concept, and have not scientific ideas and methods to solve the problem of fatigue. This paper studies elastic modulus and bending strength change rule curve of the mullite ceramic specimens under different factors in process of college undergraduate course graduation design and innovative experiments, and determines the thermal shock damage degree of ceramics materials, and then cultivate students the research ideas and research methods of fatigue failure[6-10].

\section{Experiment Method}

The Experimental Scheme. The specimen made by high temperature solid method, the size of the test piece for $10 \times 20 \times 100 \mathrm{~mm}$. Experiment includes thermal shock test and mechanical properties test.

Design of experiments was shown in Table 1. 
Table 1 Thermal shock experiment

\begin{tabular}{|c|c|c|c|c|}
\hline Test method & \multicolumn{2}{|c|}{ Elastic modulus } & \multicolumn{2}{|c|}{ Bending break strength } \\
\hline \multirow[b]{2}{*}{$\begin{array}{l}\text { A thermal } \\
\text { shock test }\end{array}$} & $\begin{array}{c}\text { Temperature } \\
\text { difference }\end{array}$ & cycles & $\begin{array}{l}\text { Temperature } \\
\text { difference }\end{array}$ & cycles \\
\hline & $\begin{array}{c}0, \quad 200^{\circ} \mathrm{C} 、 \\
400^{\circ} \mathrm{C} \cdots 1200^{\circ} \mathrm{C}\end{array}$ & Air cooling one time & $\begin{array}{c}0,100^{\circ} \mathrm{C} 、 \\
200^{\circ} \mathrm{C} \cdots 900^{\circ} \mathrm{C}\end{array}$ & $\begin{array}{c}\text { Air cooling and } \\
\text { water cooling each } \\
\text { one time }\end{array}$ \\
\hline \multirow{4}{*}{$\begin{array}{l}\text { Thermal } \\
\text { fatigue test }\end{array}$} & $\begin{array}{l}\text { Temperature } \\
\text { difference }\end{array}$ & cycles & $\begin{array}{l}\text { Temperature } \\
\text { difference }\end{array}$ & cycles \\
\hline & $400^{\circ} \mathrm{C}$ & Air cooled 60 times & $500^{\circ} \mathrm{C}$ & Air cooled 40 times \\
\hline & $600^{\circ} \mathrm{C}$ & Air cooled 60 times & $800^{\circ} \mathrm{C}$ & Air cooled 40 times \\
\hline & $800^{\circ} \mathrm{C}$ & Air cooled 45 times & & \\
\hline
\end{tabular}

Determination of Elastic modulus. Elastic modulus is measured by ultrasonic method under the condition of air cooling. Wave velocity measurement is shown in Fig.1.

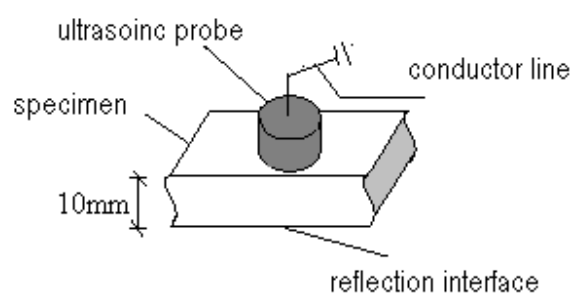

Fig. 1 Ultrasonic wave velocity measurement schematic diagram

Formula of wave velocity is that ultrasonic wave velocity is equal to (walk path of the ultrasound in material) divide by (probe emission and receiving of ultrasonic time interval).

The elastic modulus of materials is calculated according to the formula (1)[11] :

$$
E=\rho c_{T}^{2}\left[3-\frac{1}{\left(c_{L} / c_{T}\right)^{2}-1}\right]
$$

Where ${ }^{C_{L}}$ is the velocity of longitudinal waves $(\mathrm{m} / \mathrm{s}),{ }^{C_{T}}$ is the velocity of shear waves $(\mathrm{m} / \mathrm{s}), \rho$ is density.

Determination of Flexural Strength. Bending strength is determined by three point bending method, which is according to the provisions of GB3001-82. Measuring instrument is the electronic universal testing machine, and the loading rate is $0.5 \mathrm{~mm} / \mathrm{min}$. The three point bending method is shown in Fig.2. The flexural strength is calculated according to the formula (2):

$$
\sigma=\frac{3 P L}{2 B W}
$$

where $P$ is the load of fracture (N), $L$ is the distance of the two supports (mm), $W$ is the width of specimen (mm) and $B$ is the height of the specimen (mm).

\section{Result and Discussion.}

Elastic properties results and analysis.

Fig. 3 was the elastic modulus variation curve with thermal shock temperature in air cooling condition. With increasing of the thermal shock temperature difference, the value of the elastic modulus decreased greatly. Fig. 4 was elastic modulus changing curve with the cycles of thermal shock. It can be seen that with the increase of thermal shock cycles, the trend of elastic modulus was decreasing in a ladder shaped. 


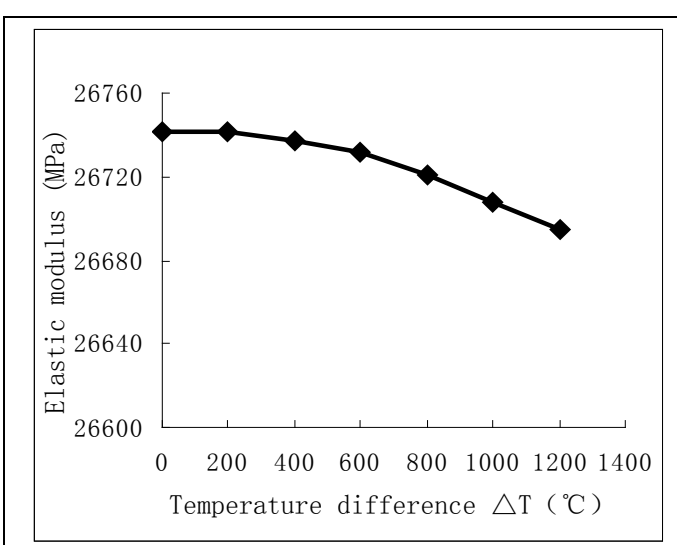

Fig.3 The elastic modulus variation with thermal shock temperature

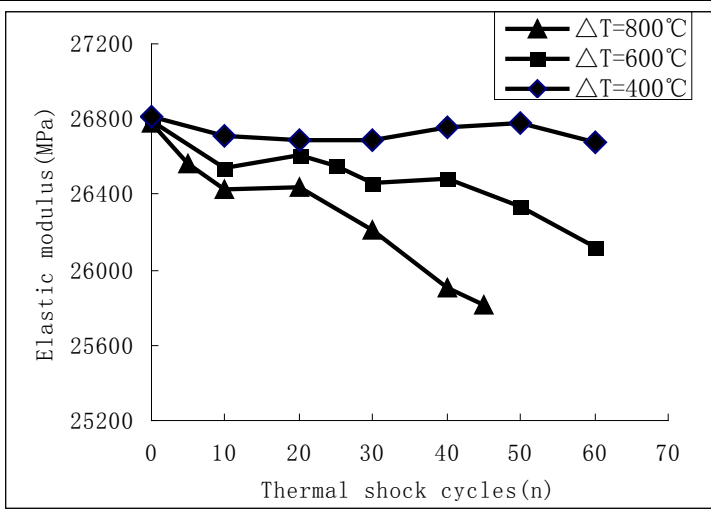

Fig. 4 The elastic modulus changes with the number of thermal shock

Fracture performance results and analysis. Under the condition of air cooling and water cooling, the bending strength change rules of one thermal shock are analyzed, respectively.

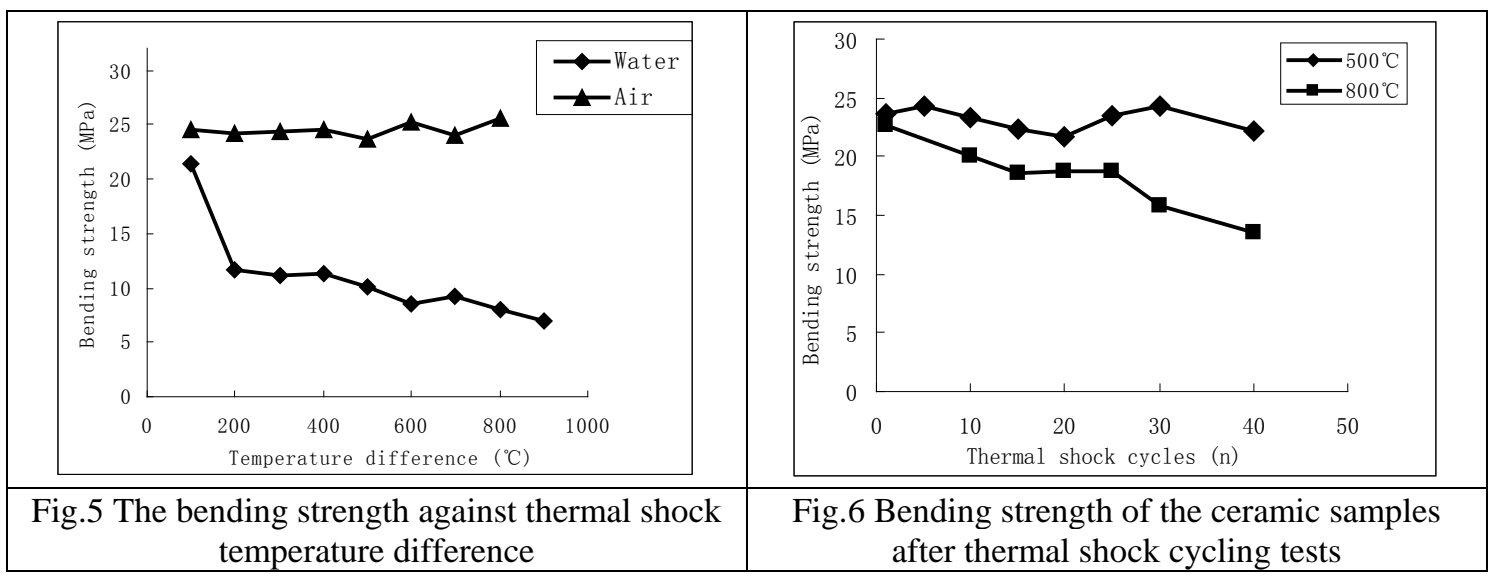

The bending strength change curves along with temperature difference in conditions of different cooling medium were shown in Fig.5. The figure showed: using air and using water as cooling medium, the bending strength variation trend is different. The change rule of bending strength along with the thermal shock cycles is displayed in Fig.6. As shown in Fig.6, the two curves of bending strength of difference temperature difference declined gradually with the increasing of thermal shock numbers.

\section{Summary}

Through experiment the change value curve of the elastic modulus and bending strength which were under different temperature, different thermal shock cycles and different cooling mediums were measured, and the damage degree of thermal shock mechanical properties on the mullite ceramics were obtained.

In the current engineering mechanics course, the fatigue, especially the thermal fatigue, almost is not involved due to the limitation of school hours, The students almost have not fatigue failure concept, and have not scientific ideas and methods to solve the problem of fatigue. But in process of college undergraduate course graduation design and innovative experiments, by studying elastic modulus and bending strength change rule curve of the mullite ceramic specimens, the students research ideas and research methods of fatigue failure could be cultivated.

\section{Acknowledgement}

This work was financially supported by the excellent course in Shandong Province "Engineering Mechanics”, a grant from National High Technology Research and Development Program of China 
(2009AA063202) and the Shandong Province Natural Science Fund (ZR2009FQ023, ZR2011EL017, ZR2013EEQ008).

\section{References}

[1]Zhao guangyan, Rao Pinggen, Lu Ming. Reserch and application of mullite and porous mullite[J], China Ceramics,2006,42(9):13-17.

[2]Chen Bei, Ding Peidao, Cheng Chuan, Zhou Zehua. Study of thermal shock resistance for ZrO2 laminated ceramics by indentation method[J],NaiHuo CaiLiao,2004,38(4),234-237

[3]Osterstock F. Contact damage submitted to thermal shock :a method to evaluate and simulate thermal shock resistance to brittle materials[J]. Mater Sci Eng,1993,A168:41-44

[4]Faber K T, Huang M D, Evans A G. Quantitative studies of thermal shock in ceramics based on a novel test technique[J]. J AmCeramSoc,1981, 64(5):296-301

[5]Li Jiake, Zhou Jianer, Liu Xin.A study on thermal-shock resistance for high-temperature oxidation resistant ceramic coating on FeCrA-ALLOY[J],Journal of Ceramics,2010,31(1):101-104

[6]Li Tingting. Preparation, characterization and thermal shock resistance of Spodumene ceramics[D]. Wuhan: Wuhan institute of technology, 2012.

[7]Lu Jun, Zheng Zhixiang, Jin Zhihao. R-curve behavior and thermal shock resistance for AL2O3 ceramic matrix composites[J], Material Composite Science ,2001,18(4),76-81

[8]Chen Bei, Ding Peidao, Cheng chuan, Zhou Zehua. Crack growth resistance behavior and thermal shock resistance for Zr02+AL2O3 laminated ceramics[J], Journal of the Chinese Ceramic Society, 2004,32(6),718-722.

[9]Andersson Tomas, Rondiffe David J. Indentation thermal shock test for ceramics[J], Am Cerm Soc,1996,79(6)

[10]Lu Jun, Zheng Zhixiang, Ling Zhihao, Ding Houfu. Indentation-quench method to determine the thermal shock resistance for toughing AL2O3 ceramic matrix composites[J], PTCA, 2003,39(1),14-18

[11]Li Jiawei. NDT handbook[M], Beijing: China Machine Press,2012. 